\title{
Radiographic interpretation by nurse practitioners in a minor injuries unit
}

\author{
R M Freij, T Duffy, D Hackett, D Cunningham, J Fothergill
}

\begin{abstract}
Objective-To compare nurse practitioners with senior house officers (SHOs) for their ability to request and interpret correctly a limited range of $x$ ray views of patients attending a minor injuries unit.

Design-Retrospective analysis of case records.

Methods-150 accident and emergency (A\&E) records with $x$ ray requests were randomly selected from the SHOs' first, second, and third 2-month period of their 6-month appointments; 150 record cards were randomly selected from a nearby minor injuries unit over the same period. Copies of the records were reviewed blind and a decision made as to whether $x$ ray requests were appropriate; $x$ ray interpretation was compared with that of a consultant radiologist.

Results $-106 x$ rays were taken on the MIU patients $(71 \%)$ and 124 on the $A \& E$ patients $(83 \%)$. There was no statistically significant differences in the ability of the nurse practitioners and the SHOs to request and interpret appropriate $x$ rays. In both groups the decision to carry out an $x$ ray was considered appropriate in $70 \%$ of patients; $\boldsymbol{x}$ rays were positive in about one third. The sensitivity of radiological interpretation was $93 \%$ in both groups, and there were $2 \%$ missed positives.

Conclusions-Appropriately trained nursae practitioners are at least as good as SHOs in recognising the need for an $x$ ray and are as competent in their interpretation. ( $f$ Accid Emerg Med 1996;13:41-43)
\end{abstract}

Key terms: accident and emergency; senior house officer; nurse practitioner; minor injuries unit; $x$ ray.

On 1st February 1993 the Accident and Emergency (A\&E) Department of St Charles's Hospital in west London was changed to a nurse managed minor injuries unit (MIU). The MIU was set up under the nursing directorate following close liaison between the $\mathrm{A} \& \mathrm{E}$ directorate, orthopaedic surgery, radiology, and other relevant departments.

The MIU is staffed by six nurse practitioners who work completely independently of doctors and is open 12 hours per day seven days per week.

The nurse practitioners underwent an intensive training programme before starting work in the MIU although many had several years' experience in A\&E work.

Protocols were drawn up which allowed the nurse practitioners to initiate investigations and treatment for a wide range of minor injuries in adults and in children over the age of 10 years.

The nurse practitioners were able to request $x$ rays within set guidelines for injuries distal to elbows and knees as well as soft tissue views for foreign bodies.

The A\&E senior house officers (SHOs) and the nurse practitioners attended a one day course on radiology; the SHOs also received a further eight hours' radiology teaching during their six month appointment. The nurse practitioners were trained in $x$ ray interpretation by a consultant radiologist.

The red dot system ${ }^{1}$ and priority next day reporting of all $x$ rays were two safeguard mechanisms which were put in place at the MIU, as was already the established procedure in the $\mathrm{A} \& \mathrm{E}$ department. ${ }^{2}{ }^{3}$ The $x$ ray interpretation by both the nurse practitioners and the SHOs was entered on the $x$ ray request form. The radiologist reporting the films then stated "agree" or "disagree", thereby facilitating early identification of errors.

The nurse practitioners work independently of the A\&E department at St Mary's Hospital which is two miles away, but have open access to second opinions from $\mathrm{A} \& \mathrm{E}$ consultants, senior registrars, and junior registrars for patients whose conditions fall outside the MIU protocols.

St Mary's Hospital has a major A\&E department to which 27634 new patients presented during the study period; 3498 patients were treated in the MIU during the same period.

There are no published studies of nurse practitioners working independently of doctors, requesting and interpreting a limited range of $x$ rays on patients attending an MIU. This study was carried out to compare the nurse practitioners with SHOs in A\&E medicine.

\section{Methods}

The nurse practitioner protocols allow for $x$ rays to be requested for injuries distal to the knees and elbows. Therefore records of patients with injuries limited to these areas were selected from the A\&E at St Mary's Hospital so that the groups were comparable. The lower age limit for inclusion in the study was 10 years, as the MIU protocols do not extend below that age.

Fifty $A \& E$ records were randomly selected from the SHOs' first, second, and third two month periods of their six month appointment from August 1993 to January 1994; 150 cards were randomly selected from the MIU over the same period. 


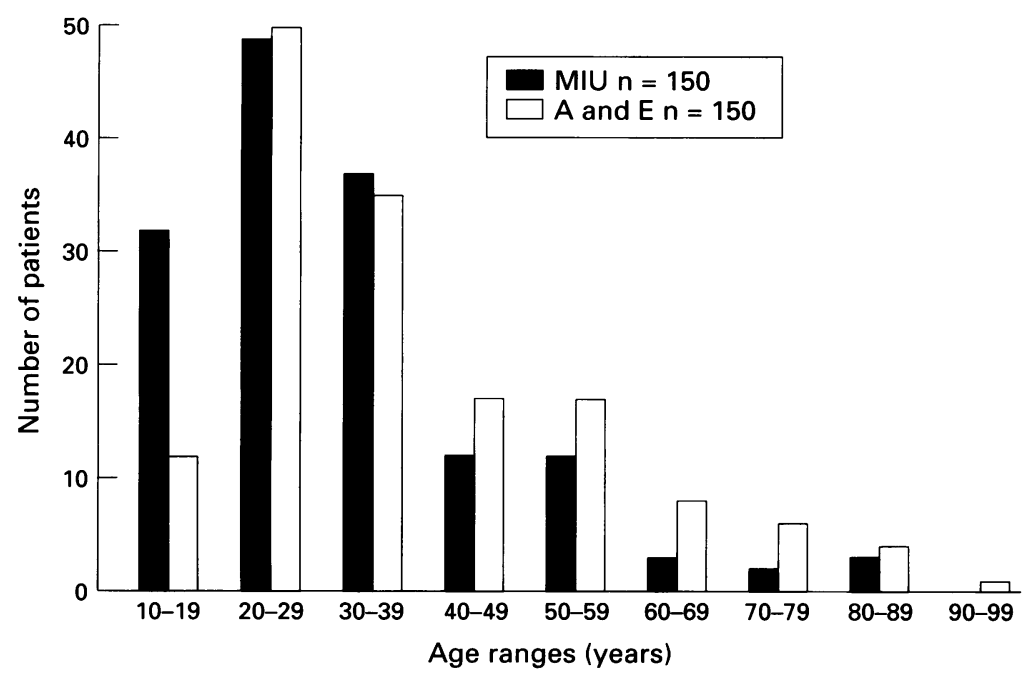

Fig 1 Age distribution

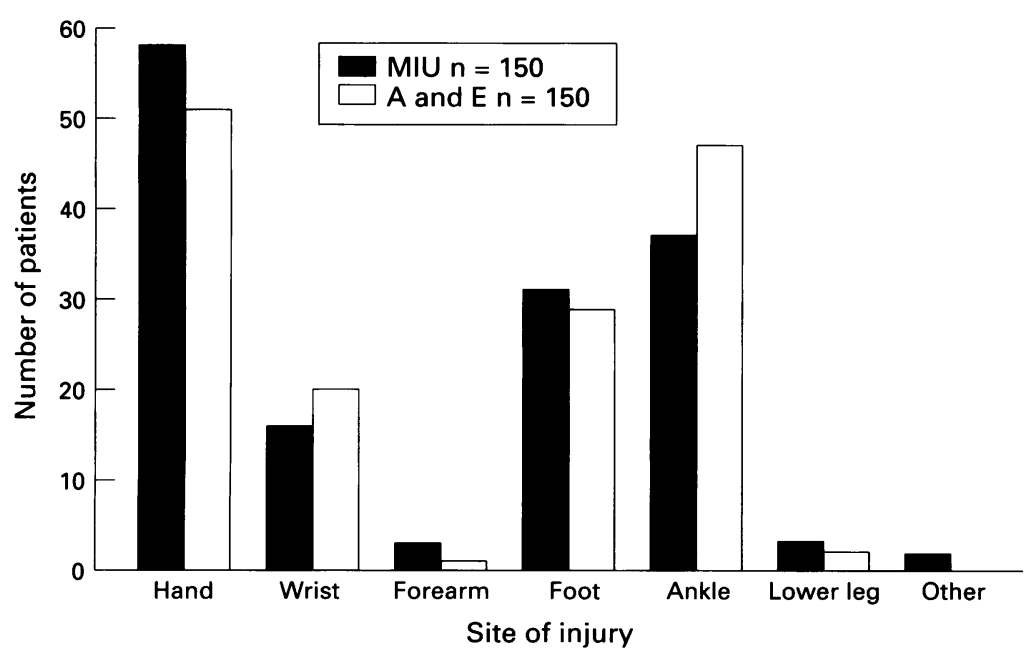

Fig 2 Site of injury

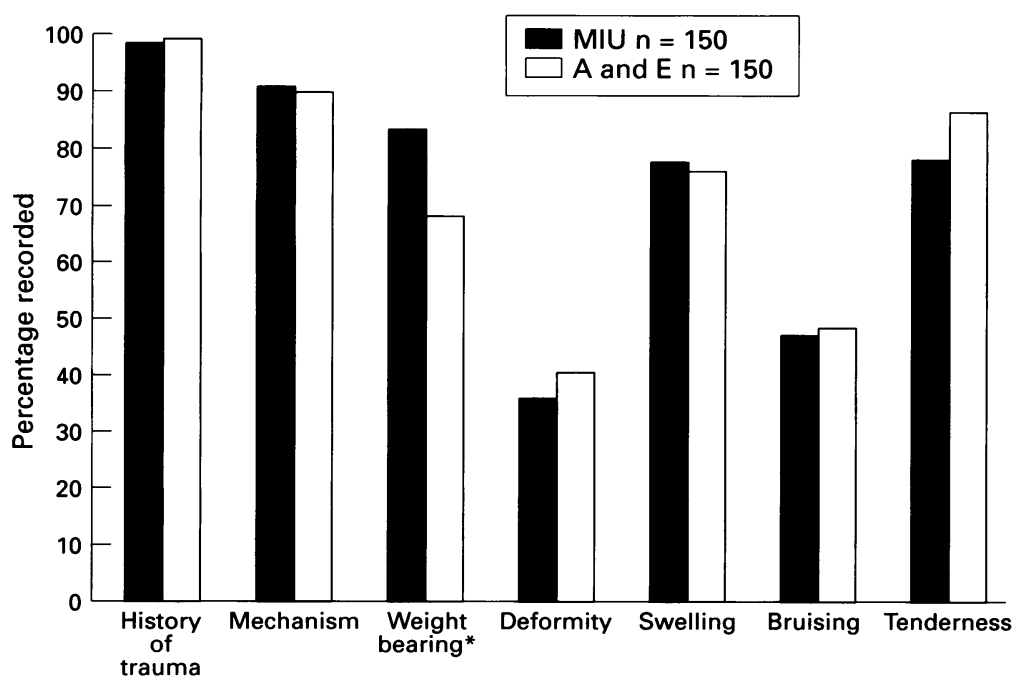

Fig 3 Clinical documentation appropriate or inappropriate on the basis of clinical information, regardless of the final $x$ ray result.

The $x$ ray interpretation was then compared with that of a consultant radiologist, which was taken as the gold standard.

The results were analysed using a $\chi^{2}$ test and the specificity and sensitivity of $x$ ray interpretation by staff at the MIU and the A\&E department were assessed.

\section{Results}

Figure 1 shows the age distribution of the patients in the MIU and A\&E patient groups. The average ages were 30 years and 37 years respectively. There were 29 children aged 10 to 16 years in the MIU group, and five in the $A \& E$ group. The male:female ratio was 49:51 in the MIU and 46:54 in the A\&E department.

The anatomical sites of injuries were similar in patients in the MIU and the A\&E department groups as indicated by fig 2 . Figure 3 shows the percentage of documented aspects of the history and clinical examination which were thought to be relevant to the injuries.

The total number of $x$ rays taken was 106 in the $150 \mathrm{MIU}$ patients $(71 \%)$ and $124(83 \%)$ in the $150 \mathrm{~A} \& \mathrm{E}$ patients. Table 1 shows the number of appropriate and inappropriate decisions to $x$ ray undertaken by the MIU nurses and the $A \& E$ department doctors. The total number of appropriate decisions was $76 \%$ in the MIU and $70 \%$ in the A\&E department. There was no statistically significant difference between the A\&E SHOs and the MIU nurse practitioners in correctly deciding whether to request an $x$ ray. A $\chi^{2}$ test was carried out on the total results for the six month period, as the numbers for each two month of the SHOs' appointment period were too small for satisfactory analysis.

Table 2 shows the number of positive and negative $x$ rays with both false negative and false positive rates. The percentage of positive $x$ rays for the MIU was $31 \%(33 / 106)$ and for A\&E 35\% (44/124).

The sensitivity of the nurse practitioners' radiological interpretation was $93.9 \%(31 / 33$; $95 \%$ confidence interval (CI) $79 \cdot 8 \%$ to $99 \cdot 3 \%$ ) and that of the A\&E SHOs was $93.2 \%$ (41/44; $95 \%$ CI $81 \cdot 3 \%$ to $98 \cdot 6 \%$ ). The specificity for $x$ ray interpretation was $93 \cdot 2 \%(68 / 73)$ for the MIU (95\% CI $84 \cdot 7 \%$ to $97 \cdot 7 \%$ ) and $92 \cdot 5 \%$ (74/80; $95 \%$ CI $84.4 \%$ to $97 \cdot 2 \%$ ) for $A \& E$ SHOs.

\section{Discussion}

There have been no previous published studies where nurse practitioners were compared directly with A\&E SHOs. The only other MIU which has recently started allowing nurse practitioners both to request $x$ rays and to interpret them independently is at St Albans City Hospital.

The results of this study show that there is no statistically significant difference between the A\&E SHOs and the MIU nurse practitioners in their ability to request and to interpret $x$ rays within a limited remit $(P>0.05)$.

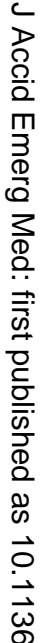 . .


Table 1 Decision to take $x$ ray

\begin{tabular}{|c|c|c|c|c|c|c|}
\hline \multirow[t]{2}{*}{ Group } & \multicolumn{2}{|c|}{ First 2 months } & \multicolumn{2}{|c|}{ Second 2 months } & \multicolumn{2}{|c|}{ Third 2 months } \\
\hline & $M I U$ & $A \mathcal{E} E$ & $M I U$ & $A \mathcal{G} E$ & $M I U$ & $A \mathcal{E} E$ \\
\hline $\begin{array}{l}X \text { ray appropriate } \\
X \text { ray inappropriate } \\
\text { Inadequate information }\end{array}$ & $\begin{array}{r}41(82 \%) \\
9(18 \%)\end{array}$ & $\begin{array}{l}33(66 \%) \\
17(34 \%)\end{array}$ & $\begin{array}{c}35(70 \%) \\
13(26 \%) \\
2(4 \%)\end{array}$ & $\begin{array}{c}32(64 \%) \\
15(30 \%) \\
3(6 \%)\end{array}$ & $\begin{array}{c}38(76 \%) \\
11(22 \%) \\
1(2 \%)\end{array}$ & $\begin{array}{l}40(80 \%) \\
8(16 \%) \\
2(4 \%)\end{array}$ \\
\hline Total & $50(100 \%)$ & $50(100 \%)$ & $50(100 \%)$ & $50(100 \%)$ & $50(100 \%)$ & $50(100 \%)$ \\
\hline
\end{tabular}

MIU, minor injuries unit; A\&E, accident and emergency.

Missed positive $x$ ray findings constituted $2 \cdot 0 \%$ of the total $x$ rays performed in the MIU and $2 \cdot 4 \%$ of the total in A\&E. This correlates well with previous published data on SHOs' ability to interpret $x$ rays. ${ }^{4}$

The number of $x$ ray requests may have been reduced further had the Ottawa ankle rules been applied, ${ }^{5}$ as ability to weight bear was not documented in $50 \%$ of relevant $A \& E$ injuries and in $20 \%$ of relevant MIU injuries.

The study confirms the previously published findings that appropriately trained nurse practitioners are at least as good as A\&E SHOs in recognising the need for an $x$ ray. ${ }^{6}$ We have also shown that they are as competent in the interpretation of $x$ rays.

Table 2 Correlation with radiologist's interpretation. Bold numbers indicate agreement

\begin{tabular}{|c|c|c|c|c|}
\hline \multirow[t]{3}{*}{ MIU or $A \mathcal{E} E$ interpretation } & \multicolumn{4}{|c|}{ Radiologist's opinion } \\
\hline & \multicolumn{2}{|c|}{ Fracture } & \multicolumn{2}{|c|}{ No fracture } \\
\hline & $M I U$ & $A \mathcal{E} E$ & $M I U$ & $A \mathcal{E} E$ \\
\hline $\begin{array}{l}\text { Fracture } \\
\text { No fracture }\end{array}$ & $\begin{array}{r}31 \\
2\end{array}$ & $\begin{array}{r}41 \\
3\end{array}$ & $\begin{array}{r}5 \\
68\end{array}$ & $\begin{array}{r}6 \\
74\end{array}$ \\
\hline Totals & 33 & 44 & 73 & 80 \\
\hline
\end{tabular}

MIU, minor injuries unit; A\&E, accident and emergency.
As $A \& E$ services are centralised there may be a place for MIUs in the community, continuing to offer a service to patients who fall within certain protocol guidelines. The St Charles' model of $\mathrm{MIU}^{7}$ is unusual, as elsewhere the majority of nurse practitioners still work within an $A \& E$ department. However, the results of our study should encourage more nurse-led MIUs to be set up with close affiliations to their parent $\mathrm{A} \& \mathrm{E}$ departments.

1 Berman L, de Lacey G, Twomey E, et al. Reducing errors in the accident department: a simple method using radiographers. Radiography 1986; 52: $143-4$.

2 Touquet $R$, Fothergill J, Harris $N$ H. Accident and emergency departments; the speciality of accident and emergency medicine. In: Powers S, Harris N H, eds. Medical negligence, 2nd ed. London: Butterworths, 1994.

3 Touquet $\mathrm{R}$, Driscoll $\mathrm{P}$, Nicholson D. The Ten commandments of Accident and Emergency Radiology. BMF 1995; 310: 642-5.

4 Vincent $C$, Driscoll $P$, Audley $R$, et al. Accuracy of detection of radiographic abnormalities by junior doctors. Arch Emerg Med 1988; 5: 101-9.

5 Steill I G, Douglas McKnight R, Greenberg G H, et al. Implementation of the Ottawa Ankle Rules. FAMA 1994 ; 271: 827-9.

6 Macleod A J, Freeland P. Should nurses be allowed to request $\mathrm{X}$-rays in an accident and emergency department? request X-rays in an accident and

7 Baker B. Model methods. Nursing Times 1993; 89 (47) 33-5. 